inaccessible stricture (treated with balloon dilatation) and 1 had no apparent luminal stenosis. Fifteen SEMS were placed for 14 patients. Stented patients had median 1 (range 0 to 6) prior surgery. Eleven patients had had prior right hemicolectomy, while 3 had ileal resection only. Attempted SEMS placement was successful in $100 \%$ of cases but could not be attempted in one case. Three adverse events were noted. There were 2 patients admitted for abdominal pain, with pain resolving upon stent removal. There was a single asymptomatic stent migration. There were no bleeding events, perforations or any need for emergency surgery. On extended follow up $(n=11) 9$ of 11 patients reported symptom resolution or improvement. To date none of the patients $(n=14)$ has required surgical intervention during follow up, with a single patient electing for re-stenting. (Ref. Figure 1)

Conclusions In this series, removable SEMS therapy for Crohn's ileocolonic strictures was effective both endoscopically and in relieving symptoms. The absence of perforations appears favourable when compared to rates reported with endoscopic balloon dilatation though a larger controlled study would be needed to test this finding. Observed long term benefit, a low re-intervention rate and no need for surgery during follow up in this series is notable. Safety and comparative efficacy against EBD should be further established with Randomised Control Trial evidence.

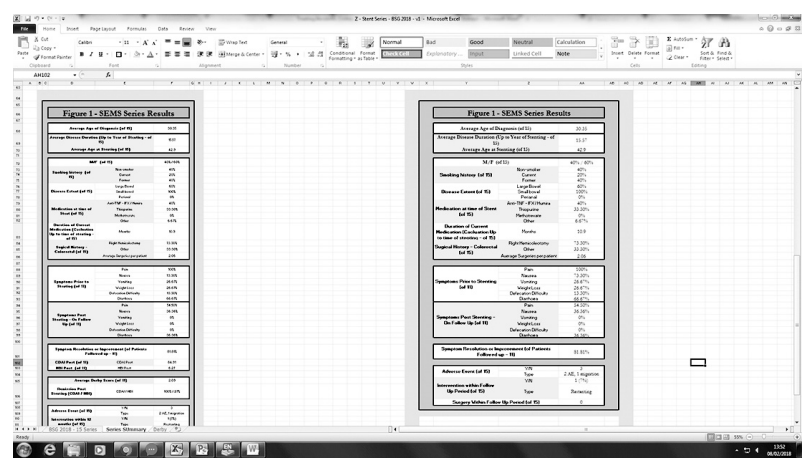

Abstract OTU-007 Figure 1

\section{OTU-008 GROWTH IN PARTICIPATION, REGISTRATIONS AND DATA MATURITY IN THE UK IBD REGISTRY: FOCUS ON BIOLOGICS}

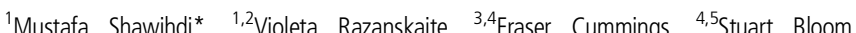

1,2Keith Bodger, Keith Bodger*. 'University of Liverpool, Liverpool, UK; ${ }^{2}$ Aintree University Hospital NHS Trust, Liverpool, UK; ${ }^{3}$ University Hospitals Southampton, Southampton, UK; ${ }^{4}$ UK IBD Registry; ${ }^{5}$ University College London Hospital, London, UK

\subsection{6/gutjnl-2018-BSGAbstracts.111}

Introduction The UK IBD Registry (IBD-R) provides a standardised dataset and alternative methods for local teams to record structured electronic data in routine care. The infrastructure enables collection of data for local use and upload of anonymized data centrally (each quarter). Registry participation allows sites to demonstrate engagement with national audit (e.g. Biologics Audit, listed on the NHS Quality Accounts) and access to centrally-developed analytics and reporting.

Methods To evaluate growth in establishment of local biologics registers using the IBD-R, we analysed three uploads of data (March, June and Sept 2017) with a focus on key data items required for producing site-level reports for patients treated with these agents (from a basic to more granular-level detail, using the 'biologics events' dataset). We generated Quarterly Reports and distributed to centres, seeking feedback to inform future iterations.

Results Participating centres grew by 59\% (32 to 51) and number of sites contributing biologics events more than doubled (16 to 37). Total registered patients in the IBD-R increased from 24633 to 31613 .

Biologics Events For adults with CD, submitted initiation events increased $>6$ fold (472 to 3126 patients), post-induction review $\sim 3$ fold $(709$ to 2,022$)$ and 12 month review $>19$ fold (22 to 437). Agents used for adult CD [UC]: Remicade 1423 [450]; Humira 1675 [311]; Inflectra 345 [236]; Remsima 398 [225]; Vedolizumab 186 [201]; Ustekinumab 16 [0]. Data completeness varied by item, e.g. recording of 'naïve' status ( $\mathrm{y}$ or $\mathrm{n}$ ) was static at $\sim 50 \%$; categorization of clinical indication consistently high at $>80 \%$ of cases.

Outcome measures By Sept 17, Physician Global Assessment (PGA) was reported at 1053 initiation events for CD (62\%) and UC 940 (66\%); and at 1537 (97\%) and 1248 (80\%) post-induction reviews showing rapid adoption, but data for disease activity indices (HBI or SCCAI) remained static at around 10\%. The next upload (Feb 2018) contains almost 40000 records from 63 sites and confirms continuing growth (analysis in progress).

Conclusions There has been significant increase in participation and in the breadth and depth of data being submitted to the UK IBD-R, particularly for biological therapies. Patterns of outcome data collection suggest clinical teams favour simple global outcome measures to formal activity indices - likely reflecting the added burden of administering and recording the data. However, the feasibility of site-level reporting to support local biologics registries is now established.

Funded by Crohn's and Colitis UK

\section{OTU-009 MODELLING CASELOAD STANDARDS FOR IBD SPECIALIST NURSES IN THE UK}

Isobel Mason* , Alison Leary. Crohns And Colitis UK, St Albans, UK

\subsection{6/gutjnl-2018-BSGAbstracts.112}

Introduction The national standards for IBD care defined the numbers of nurse specialists required as 1.5 FTE per 250000 population. The aim was to publish a new, robust, validated national standard and caseload.

Methods A concensus workshop of 15 IBD nurse specialists from across the UK met to check assumptions regarding workload and activity of this group. A 24-item questionnaire, exploring demographic data, caseload, workload and experience was developed. This was was distributed through the RCN IBD Nursing Network. Data was modelled using descriptive statistics and pattern recognition.

Results 164 responses were received (55\% response rate). $76 \%$ were from England. Responses were received from all four countries of the U.K. Most respondents covered a single $(60 \%)$ or two $(25 \%)$ hospital sites. $38 \%$ of respondents had less than 3 years experience working with IBD patients. $62 \%$ having four years plus experience. $32 \%$ had over ten years' experience. $90 \%$ of the responding CNS were working solely in IBD. $82 \%$ reported spending $80 \%$ to $100 \%$ of their time on IBD. $51 \%$ worked with adult and transition patients. $72 \%$ 